\title{
On Malcev algebras nilpotent by Lie center and corresponding analytic Moufang loops.
}

\author{
Alexander Grishkov, Marina Rasskazova, \\ Liudmila Sabinina, Mohamed Salim
}

May, 2020

\begin{abstract}
In this note we describe the structure of finite dimensional Malcev algebras over a field of real numbers $\mathbb{R}$, which are nilpotent module its Lie center. It is proved that the corresponding analytic global Moufang loops are nilpotent module their nucleus.
\end{abstract}

Key words: Malcev algebras, Moufang loops, Global Moufang loops.

2010 Mathematics Subject Classification: 17D10, 20 N05.

\section{Introduction}

The theory of analytic loops started with the work of A.I. Malcev in [Ma1]. In this article, the correspondence between the analytic local diassociative loops and the binary Lie algebras was established. A loop is a set $M$, endowed with a binary operation $M \times M \rightarrow M$, with the neutral element $e \in M$ and the condition that the equations $a x=b, y a=b$ for all $a, b \in M$ have a unique solution. A loop is called diassociative, if every two elements of this loop generate a subgroup. Malcev showed that for an analytic loop, with the Moufang identity: $(x y)(z x)=x(y z) x$ the corresponding tangent algebra satisfies the following identities:

$$
x^{2}=[J(x, y, z), x]-J(x, y,[x, z])=0,
$$

where $J(x, y, z)=[[x, y], z]+[[x, z], x]+[[z, x], y]$ (See [1]). Algebras with these defining identities are currently called Malcev algebras. The more difficult question, if every finite dimensional Malcev $\mathbb{R}$-algebra is the tangent algebra of some local analytic Moufang loop was solved positively by E.Kuzmin in 1969 in [Kuzm2].

Let us consider a pair $(\mathfrak{a}, \mathfrak{L})$, where $\mathfrak{a}$ is some subvariety of binary Lie algebras (in particular Malcev algebras) and $\mathfrak{L}$ is a subvariety of diassociative loops (in particular, Moufang loops).

A pair $(\mathfrak{a}, \mathfrak{L})$, will be called locally dual, if it satisfies the following conditions: 
- every tangent algebra of a local analytic loop from the variety $\mathfrak{L}$ belongs to the variety $\mathfrak{a}$,

- every finite-dimensional $\mathbb{R}$-algebra from the variety $\mathfrak{a}$ is a tangent algebra of some local analytic loop from the variety $\mathfrak{L}$

The description of all locally dual pairs is a meaningful and difficult problem.

If a locally dual pair $(\mathfrak{a}, \mathfrak{L})$ satisfies a stronger condition, namely, if for every global analytic loop $S$ such that its local loop belongs to $\mathfrak{L}$, is in $\mathfrak{L}$ too, we will call such a pair $(\mathfrak{a}, \mathfrak{L})$ globally dual.

Kerdman $\overline{K e r}$ showed that a pair $(\mathfrak{m}, \mathfrak{M})$ is globally dual if $\mathfrak{m}$ is the variety of all Malcev algebras, and $\mathfrak{M}$ is a variety of all Moufang loops. In this note we study the duality of a pair formed by two varieties: the first one, $N L_{k}$, is the subvariety of the variety $\mathfrak{m}$, which is defined by the identity $J\left(\left[x_{1}, x_{2}, \ldots, x_{k}\right], y, z\right)=0$, where in the product $\left[x_{1}, x_{2}, \ldots, x_{k}\right]$ a distribution of parentheses is arbitrary and the second one, $N G_{k}$, is the subvariety of the variety $\mathfrak{M}$, defined by the identity $\left(\left[x_{1}, x_{2}, \ldots, x_{k}\right], y, z\right)=1$. Here $\left[x_{1}, x_{2}, \ldots, x_{k}\right]$ is a commutator of length $k$ with an arbitrary distribution of parentheses and $(a, b, c)=((a b) c)(a(b c))^{-1}$ is an associator.

For a Malcev algebra $M$ we define $M^{1}=M, M^{n}=\sum_{i, j>0}^{i+j=n}\left[M^{i}, M^{j}\right]$. Let $F$ be a free Malcev algebra, then $N L_{k}$ is a variety of Malcev algebras defined by all identities of the type $J(w, x, y)=0$, where $w \in F^{k}, k \geq 2$.

\section{Structure of finite dimensional Malcev algebras nilpotent by Lie center.}

Let $M$ be a finite dimensional Malcev algebra over a field $\mathbb{C}$, and let $G$ be a solvable radical of $M$. Then there exists a semisimple subalgebra (Levi factor) $S$, such that $M=S \oplus G$ ( [Gri1],[Kuzm1],[Car]).

We will use the results and the terminology from [Gri2]. Let $g \in M$, the element $g$ is said to be splitting element if $g=t+n$, where $n$ is a nilpotent element and $t$ is a semisimple element, i.e., the right multiplication operator $R_{t}$ is diagonalizable and the operators $R_{t}$ and $R_{n}$ commute. A Malcev algebra $M$ is said to be splitting if all elements of $M$ are splitting. If $M$ is a finite-dimensional splitting Malcev algebra over a field of characteristic 0, then $M=S \oplus T \oplus N$, where $S$ is a semisimple Levi factor, $T$ is an abelian subalgebra of $M$ such that each element of $T$ is semisimple (toroidal subalgebra), and $N$ is the nilpotent radical of $M$. Additionally $[S, T]=0$,

$$
N=\sum_{\alpha \in \Delta} \bigoplus N_{\alpha}
$$

where $\Delta \subset T^{*}=\operatorname{Hom}_{k}(T, k)$ and

$$
N_{\alpha}=\{x \in N \mid[x, t]=\alpha(t) x, \quad \forall t \in T\} .
$$


Moreover, $\left[N_{\alpha}, N_{\beta}\right] \subseteq N_{\alpha+\beta}$, if $\alpha \neq \beta$, and $\left[N_{\alpha}, N_{\alpha}\right] \subseteq N_{2 \alpha}+N_{-\alpha}$.

Since $[T, S]=0$, one has that $N_{\alpha}$ is an $S$-module and hence $N_{0}=N_{01} \oplus N_{00}$, $\left[S, N_{0}\right]=\left[S, N_{01}\right]=N_{01}$ and $\left[S, N_{00}\right]=0$. Set

$$
M_{11}=\left(S \oplus \sum_{\alpha \in \Delta \backslash 0} \bigoplus N_{\alpha}\right) \oplus N_{01}
$$

and let us denote by $M_{1}$ the subalgebra generated by $T \oplus M_{11}$. Notice that in general, $M_{1} \neq T \oplus M_{11}$, and $\left[N_{01}, N_{00}\right] \subseteq N_{01},\left[M_{11}, N_{0}\right] \subseteq M_{11}$. Hence $M_{1}$ is an ideal. Every finite-dimensional Malcev algebra $M$ over a field of characteristic 0 is contained in some splitting Malcev algebra $\hat{M}$. If such $\hat{M}$ does not contain intermediate splitting subalgebra, which contains $M$, then $\hat{M}$ is called a splitting of $M$. Each automorphism of the algebra $M$ extends uniquely to an automorphism of a splitting of $M$. If $\hat{M}$ is a splitting of $M$, then $\hat{M}^{2}=M^{2}$, and any ideal of the algebra $M$ is an ideal of the algebra $\hat{M}$ and vice verse any ideal of $\hat{M}$, which is in $M$ is also the ideal of $M$. This result is analogous to one for Lie algebras due to A. I. Malcev [Ma2].

In what follows in this article the splitting algebra of an algebra $M$ we will denote by $\hat{M}$.

Recall that

$$
\operatorname{Lie}(M)=\{x \in M \mid J(x, M, M)=0\}
$$

is the Lie center of $M$.

Lemma 1. In this notation Lie $(M) \subseteq \operatorname{Lie}(\hat{M})$.

Proof. By the construction $\hat{M}=\bigcup_{i=1}^{n} M(i)$, where $M(1)=M, M(n)=\hat{M}$, $\operatorname{dim} M(i)=\operatorname{dim} M(i-1)+1, M(i)=M(i-1)+\mathbb{R} t_{i}, i \geq 2$, where $t_{i}$ is a semisimple element, i.e. $R_{t_{i}}: M(i-1) \rightarrow M(i-1)$ is a diagonalizable operator. Moreover there exists $x_{i} \in M(i-1)$, such that $n_{i}=x_{i}-t_{i}$ is a nil element, i.e $R_{n_{i}}$ is a nilpotent operator and $R_{t_{i}} \circ R_{n_{i}}=R_{n_{i}} \circ R_{t_{i}},\left[n_{i}, t_{i}\right]=0$.

Now consider $l \in \operatorname{Lie}(M(i-1)), x \in M(i-1)$. It is sufficient to show that $J\left(l, x, t_{i}\right)=0$. Suppose that

$$
M(i-1)=\sum_{\alpha \in \Delta} \oplus M(i-1)_{\alpha}
$$

is a Cartan decomposition with respect to the operator $R_{t_{i}}$ or $R_{x_{i}}$.

Without loss of generality one can assert that

$$
l \in \operatorname{Lie}\left(M(i-1) \cap M(i-1)_{\alpha}, x \in M(i-1)_{\beta} .\right.
$$

If $\alpha \neq \beta$, then $J\left(l, x, t_{i}\right)=0$ due to the fact that any Malcev algebra is a binaryLie algebra. 
Consider the case $\alpha=\beta \neq 0$.

The equality $J\left(l, x, t_{i}\right)=0$ is equivalent to $[x, l] \in M(i-1)_{2 \alpha}$.

Indeed, suppose that $[x, l] \notin M(i-1)_{2 \alpha}$. Then $[x, l]=[x, l]_{2 \alpha}+[x, l]_{-\alpha}$, where $[x, l]_{2 \alpha} \in M(i-1)_{2 \alpha}$ and $0 \neq[x, l]_{-\alpha} \in M(i-1)_{-\alpha}$. Recall that $x_{i}-n_{i}=t_{i}$.

$0=J\left(l, x, x_{i}\right)=\left[[x, l]_{2 \alpha},\left(t_{i}+n_{i}\right)\right]+\left[[x, l]_{-\alpha},\left(t_{i}+n_{i}\right)\right]+\left[\left[x,\left(t_{i}+n_{i}\right)\right], l\right]-\left[\left[l,\left(t_{i}+n_{i}\right)\right], x\right]$

We get

$$
\begin{gathered}
2 \alpha[l, x]_{2 \alpha}-\alpha[l, x]_{-\alpha}+\left[[x, l]_{2 \alpha}+[x, l]_{-\alpha}, n_{i}\right]+\left[\left[x, t_{i}\right], l\right]+\left[\left[x, n_{i}\right], l\right]-\left[\left[l, t_{i}\right], x\right]-\left[\left[l, n_{i}\right], x\right] \\
=2 \alpha[l, x]_{2 \alpha}-\alpha[l, x]_{-\alpha}+\left[\left[(x, l]_{2 \alpha}+[x, l]_{-\alpha}\right), n_{i}\right]+\left[\left[x, n_{i}\right], l\right]+2 \alpha\left([x, l]_{2 \alpha}+[x, l]_{-\alpha}\right) \\
-\left[\left[l, n_{i}\right], x\right]=-2 \alpha[x, l]_{2 \alpha}+\alpha[x, l]_{-\alpha}+2 \alpha\left([x, l]_{2 \alpha}+[x, l]_{-\alpha}\right)+\left[\left([x, l]_{2 \alpha}+[x, l]_{-\alpha}\right), n_{i}\right] \\
+\left[\left[x, n_{i}\right], l\right]_{2 \alpha}+\left[\left[x, n_{i}\right], l\right]_{-\alpha}-\left[\left[l, n_{i}\right], x\right]_{2 \alpha}-\left[\left[l, n_{i}\right], x\right]_{-\alpha}=0
\end{gathered}
$$

Finally we get

$$
3 \alpha[x, l]_{-\alpha}+\left[[x, l]_{-\alpha}, n_{i}\right]+\left[\left[x, n_{i}\right], l\right]_{-\alpha}-\left[\left[l, n_{i}\right], x\right]_{-\alpha}=0
$$

For every element $0 \neq a \in M(i-1)_{\alpha}$ one can define the number $|a|=\min \left\{m \mid R_{n_{i}}^{m} a=0\right\}$. Then the equality $[x, l]_{-\alpha}=0$ one can show by induction on the sum $|x|+|l|$. If $|x|+|l|=2$ one has $\left[\left[x, n_{i}\right], l\right]_{-\alpha}=\left[\left[l, n_{i}\right], x\right]_{-\alpha}=$ 0 . Now suppose that $2<|x|+|l|=m$.

Then one has $\left|\left[x, n_{i}\right]\right|+|l|<m$ and $\left|\left[l, n_{i}\right]\right|+|x|<m$ and by induction hypothesis $\left[\left[x, n_{i}\right], l\right]_{-\alpha}=\left[\left[l, n_{i}\right], x\right]_{-\alpha}=0$.

Therefore by (3) we get

$$
3 \alpha[x, l]_{-\alpha}+\left[[x, l]_{-\alpha}, n_{i}\right]=0
$$

Now if $\left|[x, l]_{-\alpha}\right|=s$ we can apply $R_{n_{i}}^{s-1}$ to the equality (4) and get $[x, l]_{-\alpha}=0$. In the case $\alpha=\beta=0$ we have $J\left(l, x, t_{i}\right)=0$, since $\left[N_{0}, N_{0}\right] \subseteq N_{0}$ and $\left[N_{0}, t_{i}\right]=0$

Thus one has

$$
\operatorname{Lie}(M) \subseteq \ldots \subseteq \operatorname{Lie}(M(i)) \subseteq \ldots \subseteq \operatorname{Lie}(\hat{M})
$$

In the theory of Lie algebras there exists the following construction of $d e-$ composable extension. Let $L$ be a Lie algebra and let $N$ be a subalgebra of the Lie algebra $\operatorname{Der} L$ (the algebra of all derivations of $L$ ). Then the direct sum $N \oplus L$ has a structure of Lie algebra with the multiplication:

$$
(a, l) \cdot(b, r)=\left([a, b], l^{b}-r^{a}+[l, r]\right) .
$$

Notice that we are not assuming that $N$ or $L$ is abelian. 
This construction has a generalization for Malcev algebras.

Suppose that $M$ is a Malcev algebra such that $M=\tilde{N}+L$, where $L \subseteq$ $\operatorname{Lie}(M)$, and $M$ has an ideal $I \in \tilde{N}$ such that $J(\tilde{N}) \subseteq I,[I, L]=0$, then $\tilde{N} / I \cong N$ is a Lie algebra. It means that $N$ acts on $L$ by derivations. In this case the formula (5) defines a Malcev algebra structure on $\tilde{M}=\tilde{N} \oplus L$, where $I$ acts trivially on the Lie algebra $L$ by definition. This construction is called the decomposable extension of Malcev algebras. Notice that in this construction $L$ is an ideal contained in the Lie center of $M$.

In what follows the decomposable extension of Malcev algebra will be denoted by $\tilde{M}$.

The aim of this section is to show the following

Theorem 1. Let $M$ be a finite dimensional Malcev algebra from the variety $N L_{k}$ over a field of complex numbers $\mathbb{C}$. Then

1. $M$ may be embedded into the splitting Malcev algebra $\hat{M}=S \oplus T \oplus N \in$ $N L_{k}$, where $S$ is a semi-simple Lie subalgebra, $T$ is a toroidal subalgebra, $N$ is a nilpotent ideal.

2. $N=N_{00} \oplus[S, N]$, where $N_{00}$ is a Malcev subalgebra of $N$ and the ideal $M_{1}$ generated by $S \oplus T \oplus\left(\sum_{\alpha \in \Delta \backslash 0} \oplus N_{\alpha}\right) \oplus[S, N]$ is contained in Lie $(\hat{M})$.

3. There exists a Malcev algebra $\tilde{M}=N_{00} \oplus M_{1}$ of the variety $N L_{k}$, which is a decomposable extension of a nilpotent Malcev algebra $N_{00}$ and a Lie subalgebra $M_{1}$, such that there exists an epimorphism $\pi: \tilde{M} \longrightarrow \hat{M}$.

In order to prove this Theorem we need to collect some intermediate results which we will present in the following lemmata.

Put $\bigcap_{n=1}^{\infty} M^{i}$ by $M^{\omega}$. Following introduced notation one has:

Lemma 2. Let $M$ be a splitting Malcev algebra from the variety $N L_{k}, k \geq 2$. Then ideal $M_{1}$, constructed above, contains $M^{\omega}$ and is contained in the Lie center $\operatorname{Lie}(M)$.

Proof. By definition of the variety $N L_{k}$ we get that $M^{\omega} \in \operatorname{Lie}(M)$ the Lie center of $M$. By construction $M_{11} \subseteq M^{\omega} \subseteq \operatorname{Lie}(M)$, hence $M^{\prime} \subseteq \operatorname{Lie}(M)$ where $M^{\prime}$ is a subalgebra of $M$ generated by $M_{11}$. It is clear that $M_{1}=T \oplus M^{\prime},\left[T, M^{\prime}\right] \subseteq$ $M^{\prime}=S \oplus V$ with $V \subseteq N$. Hence for proving the lemma it is enough to prove that $J(x, y, z)=0$, where $x, y$ and $z$ are elements of $T \cup\left(\cup_{\alpha \in \Delta} N_{\alpha}\right)$. If $x, y \in T$, then $z \in N_{\alpha}$ and

$$
J(x, y, z)=[[z, x], y]+[[(y, z)], x]=\alpha(x) \alpha(y) z-\alpha(y) \alpha(x) z=0 .
$$

If $x \in T, y \in N_{\alpha}, z \in N_{\beta}$ and $\alpha \neq 0$ or $\beta \neq 0$, then $y \in M_{11} \subseteq \operatorname{Lie}(M)$ or $z \in \operatorname{Lie}(M)$, hence $J(x, y, z)=0$. At last, in the case $\alpha=\beta=0$ we get $J(x, y, z)=0$ since $\left[N_{0}, N_{0}\right] \subseteq N_{0}$. 
Lemma 3. Let $M$ be a Malcev algebra from the variety $N L_{k}$.

Then $\left[J(M), M^{\omega}\right]=0$.

Proof. By the result of Filippov (see [Fi],page 236) one has $[J(M), \operatorname{Lie}(M)]=0$. On the other hand, $M^{\omega} \subseteq \operatorname{Lie}(M)$ since $M \in N L_{k}$.

Since $M_{1} \subseteq M^{\omega}$ by Lemma 3 we have $\left[J(M), M_{1}\right]=0$. Then the subalgebra $N_{00}$ acts on the ideal $M_{1}$ by derivations, hence it is possible to construct, as above, a Malcev algebra $\tilde{M}=N_{00} \oplus M_{1}$ with a product given by (5).

It is easy to see that the morphism $\varphi: \tilde{M} \longrightarrow M, \quad \varphi(n, m)=n+m$ is an epimorphism of Malcev algebras.

Lemma 4. The Malcev algebra $\tilde{M}=N_{00} \oplus M_{1}$ with the product given by (5) is a Malcev algebra of the variety $N L_{k}$.

Proof. Since $M_{1} \subset \operatorname{Lie}(\tilde{M})$, one has $J(\tilde{M})=J\left(N_{00}\right)$. By construction, $\left[M_{1}, J\left(N_{00}\right)\right]=0$. In this case, $\tilde{M}$ is a Malcev algebra of the variety $N L_{k}$ if and only if $N_{00}$ is a Malcev algebra of the variety $N L_{k}$; which is exactly our case.

Remark. In general, if we have a Malcev algebra $P=P_{0}+P_{1}$, where $P_{0}$ is a nilpotent subalgebra, $P_{1} \subseteq \operatorname{Lie}(P)$ is an ideal contained in the Lie center of $P$ and $P / P_{1}$ is a Malcev algebra of the variety $N L_{k}$, then $P$ is not necessarily a Malcev algebra of the the variety $N L_{k}$. It is possible that $P \in N L_{k+1} \backslash N L_{k}$.

Example. Set $P_{1}=\mathbb{R}\{t, a, b, c \mid[a, t]=a,[b, t]=-b,[a, b]=c,[c, t]=$ $[a, c]=[b, c]=0\}$ and let it be a splitting Lie algebra. Choose any nilpotent Malcev algebra $P_{0}$ which is not a Malcev algebra from the variety $N L_{k}$, but $P_{0} / Z$ is. Here $Z=\mathbb{R} z$ is some central ideal of $P_{0}$. It is easy to construct an algebra with those properties. Let us consider $\tilde{P}=P_{0} \oplus P_{1}$ and $P=\tilde{P} / I$, where $I=\mathbb{R}(c-z)$ is a central ideal. It is clear that $P$ is not a Malcev algebra from the variety $N L_{k}$. But $P=\pi\left(P_{0}\right)+\pi\left(P_{1}\right)$, where $\pi: \tilde{P} \longrightarrow P$ is a canonical homomorphism. Notice that $\pi\left(P_{0}\right) \cong P_{0}, \pi\left(P_{1}\right) \cong P_{1} \subseteq N(P)$ and $P / \pi\left(P_{1}\right)=P_{0} / Z$ is a Malcev algebra from the variety $N L_{k}$.

Proof of the Theorem.

Consider $\hat{M}=S \oplus T \oplus N$ as splitting algebra of $M=S \oplus G$. We will show that $\hat{M} \in N L_{k}$ if $M \in N L_{k}$. Due to the construction of $\hat{M}$ ([Gri2]) any ideal $I \triangleleft M$ is also the ideal of $\hat{M}$ and therefore $M^{k}=\hat{M}^{k}, k \geq 2$. Now since $\operatorname{Lie}(M) \subseteq \operatorname{Lie}(\hat{M})$ and $M^{k} \subseteq \operatorname{Lie}(M)$ one has $M^{k} \subseteq \operatorname{Lie}(M) \subseteq \operatorname{Lie}\left(M^{k}\right)$. This means $M^{k} \in N L_{k}$. As it was noticed above $N$ is a semisimple $S \oplus T$ - module, therefore $N_{00}=A n n_{N}(S \oplus T)$ is the nilpotent subalgebra of $\hat{M}$. Moreover

$$
M_{11} \subseteq \hat{M}^{\omega} \subseteq \operatorname{Lie}(\hat{M})
$$

Since [ $\left.T, N_{00}\right]=0$ one has $N_{00} \subseteq N_{0}$. In other hand in general case $N_{00} \cap M_{1} \neq 0$. Recall that $M_{1}$ is the ideal generated by $T \oplus M_{11}$. Finally one gets $\tilde{M}=$ 
$N_{00} \oplus M_{1}$.

\section{Malcev algebras and global Moufang loops.}

A variety $\mathbf{M}$ of Malcev algebras will be called (locally) smooth, if there exists a variety of Moufang loops $\mathcal{L}$ such that the pair $(\mathbf{M}, \mathcal{L})$ is (locally) globally dual. Analogously, a variety of Moufang loops $\mathcal{L}$ is (locally) smooth if there exists a variety of Malcev algebras $\mathbf{M}$, such that the pair $(\mathbf{M}, \mathcal{L})$ is (locally) globally dual. A dual pair $(M, \mathcal{L})$ will be called global if for any local analytic loop $G$ of the variety $\mathcal{L}$, there exists a global analytic loop $\tilde{G}$ from the variety $\mathcal{L}$ which is locally isomorphic to $G$. It is clear, that not all varieties of Moufang loops are smooth. For example, the variety $\mathbf{B}_{n}$ of Moufang loops of exponent $n$ is not smooth, since every analytic Moufang loop of a positive dimension is not periodic. Nevertheless we have the following Conjecture:

Conjecture 1. Every dual pair $(M, \mathcal{L})$ of Malcev algebras and their corresponding Moufang loops is global.

Notice that if the pair $(\mathbf{M}, \mathcal{L})$ is locally dual and the variety $\mathbf{M}$ contains only Lie algebras, then all finite dimensional Lie algebras from $\mathbf{M}$ are solvable. Indeed, if $M \in \mathbf{M}$ is not solvable finite dimensional then $M=S \oplus G$, where $S$ is semisimple Lie subalgebra. Hence $S$ contains some simple 3 -dimensional Lie subalgebra $L$. But the corresponding Lie group $G(L)$ contains free subgroup. Hence $\mathcal{L}$ is variety of all groups and $\mathbf{M}$ is the variety of all Lie algebras.

We will prove the Conjecture 1 for the pairs $\left(N L_{k}, G_{k}\right)$, where $N L_{k}$ is the variety defined in the last section and $G_{k}$ is a variety of Moufang loops defined by all identities of the type $(w, x, y)=1$, where $w \in F^{k}, k \geq 2$ and $F$ is an infinite free generated Moufang loop such that $F^{1}=F$, and $F^{k}$ is the normal subloop generated by $\prod_{i=1}^{k-1}\left[F^{i}, F^{k-i}\right]$.

Proposition 1. The pair $\left(N L_{k}, G_{k}\right)$ is dual for any $k \geq 2$.

Proof. Let $M$ be a Malcev $\mathbb{R}$-algebra of dimension $n$ of the variety $N L_{k}$. Then $M \cong \mathbb{R}^{n}$. There exists a small ball $M_{\epsilon}=\{x \in M|| x \mid \leq \epsilon\}$, which is a local Moufang loop with the product given by the Campbell-Hausdorff formula

$$
x \cdot y:=\mathrm{CH}(x, y)=x+y+\frac{1}{2}[x, y]+\cdots .
$$

Notice that the element 0 of $M$ is the unit of this local analytic loop. From (6) we have that for every subalgebra $P$ of $M$ the corresponding local subgroup is given by $P_{\epsilon}=P \cap M_{\epsilon}$. The subgroup $P_{\epsilon}$ is normal if and only if $P$ is an ideal of $M$. 
From (6) we get

$$
\{x, y\}=x^{-1} \cdot y^{-1} \cdot x \cdot y=[x, y]+\sum_{s=3}^{\infty} a_{s}(x, y)
$$

where $a_{s}(x, y) \in M^{s}$ if $x, y \in M$. Hence every commutator $w$ in the local Moufang loop $(M, \cdot)$ of length $k \geq 3$ has the form $w=\sum_{i=k}^{\infty} w_{i}$, with $w_{i} \in M^{i}$. Since $M \in N L_{k}$, then $M^{s} \subseteq \operatorname{Lie}(M)$ for $s \geq k$. Hence the corresponding commutator subloop $M_{\epsilon}^{k}$ of local Moufang loop $M_{\epsilon}$ is contained in $\operatorname{Lie}(M)$.

E.Kuzmin proved [?], that in a local Moufang loop $(M, \cdot)$ the associator can be expressed as:

$$
(x, y, z)=\frac{1}{6} J(x, y, z)+\sum_{i=7}^{\infty} a_{i}(x, y, z)
$$

where $a_{i}(x, y, z)$ is an element of degree $i$ of the ideal $J(M) \subset M$.

By (8) we get that $\operatorname{Lie}(M) \cap M_{\epsilon} \subseteq N u c\left(M_{\epsilon}\right)$, where $N u c\left(M_{\epsilon}\right)=\{x \in$ $\left.M_{\epsilon} \mid(x, a, b)=0, \forall a, b \in M_{\epsilon}\right\}$. Hence $M_{\epsilon}^{k} \subset \operatorname{Lie}(M) \cap M_{\epsilon} \subset N u c\left(M_{\epsilon}^{k}\right)$. It means that $\left(M_{\epsilon}, \cdot\right) \in G_{k}$.

Now suppose that $\left(M_{\epsilon}, \cdot\right) \in G_{k}$. Following the previous notation, we have:

Lemma 5. $M^{k} \cap M_{\epsilon}=\left(M_{\epsilon}, \cdot\right)^{k}$, where $\left(M_{\epsilon}, \cdot\right)^{k}$ is a commutator subloop of the local loop $\left(M_{\epsilon}, \cdot\right)$ of degree $k$.

Proof. From the construction of the local loop $\left(M_{\epsilon}, \cdot\right)$, for every ideal $I$ of the Malcev algebra $M$ there is a corresponding normal subloop $I_{\epsilon}=I \cap M_{\epsilon}$ of $\left(M_{\epsilon}, \cdot\right)$. It is clear that for nilpotent of class $k$ Malcev algebra $M / M^{k}$ the corresponding local Moufang loop is $\left(\left(M / M^{k}\right)_{\epsilon}, \cdot\right)$, which is nilpotent of class $k$. Hence $\left(M_{\epsilon}, \cdot\right)^{k} \subseteq M^{k}$.

Suppose that $\left(M_{\epsilon}, \cdot\right)$ is a nilpotent local loop of class $k$. By induction we prove that the Malcev algebra $M$ is nilpotent of the same class $k$. It is clear for $k=1$. If the Malcev algebra $M$ is not nilpotent of degree $k$ then for some $x_{1}, \ldots, x_{k} \in M$ we have $w=\left[x_{1}, \ldots, x_{k}\right] \neq 0$ for some distribution of parentheses. By (77) we get in $\left(M_{\epsilon}, \cdot\right): u_{t}=\left\{t x_{1}, t x_{2}, \ldots, t x_{k}\right\}=t^{k} w+\sum_{i>k} t^{i} w_{i}$, where $w_{i}$ is an element of $M^{i}$ and $t \in \mathbb{R}$. Since $(M, \cdot)$ is nilpotent of degree $k, u=0$ in $(M, \cdot)$. Then $w=0$ in $M$ which yields to a contradiction.

With all considerations above Lemma 5 is proved.

Now we can finish the proof of Proposition 1. Let $w=\left[x_{1}, \ldots, x_{k}\right] \in M^{k}$, we have to prove that $w \in \operatorname{Lie}(M)$. For some $t \in \mathbb{R}$ we have by Lemma 5 that $t^{k} w=\left[t x_{1}, \ldots ., t x_{k}\right] \in M^{k} \cap M_{\epsilon} \subset\left(M_{\epsilon}, \cdot\right)^{k} \subset N u c\left(M_{\epsilon}\right)$. Here we used that $\left(M_{\epsilon}, \cdot\right) \in G_{k}$. Hence $\left(t^{k} w, x, y\right)=0$ for all $x, y \in M_{\epsilon}$. By 8 we get that $J\left(t^{k} w, x, y\right)=0$. It means that $w \in \operatorname{Lie}(M)$. Proposition $\square$ is proved. 
Now we are ready to prove the main result of this paper.

Theorem 2. The dual pair $\left(N L_{k}, G_{k}\right)$ is global.

Proof. Let $G_{0} \in G_{k}$ be a local analytic loop and let $L\left(G_{0}\right)=M \in N L_{k}$ be its corresponding Malcev algebra. Let $\varphi: M \hookrightarrow \hat{M}$ be an embedding of $M$ in a splitting Malcev algebra $\hat{M}=S \oplus T \oplus N$, (see notation of Theorem 1). By definition $\hat{M}$ is minimal with this property. Hence $[\hat{M}, \hat{M}]=[M, M]$. Since $M \in N L_{k}$ then $\hat{M} \in N L_{k}$ by Theorem 1. By [Ker there exists the corresponding to $\hat{M}$ global analytic simply connected Moufang loop $\hat{G}$.

By construction of $\hat{G}$ in Ker] we get that $\hat{G}=P \cdot Q$, where $P$ is simply connected semisimple Lie group with corresponding Lie algebra $S$ and $Q=Q_{0} \cdot Q_{1}$ is simply connected solvable Moufang loop with corresponding Malcev algebra $T \oplus N$, and $Q_{0} \simeq \mathbb{R}^{t} \simeq T$ is abelian vectorial group Lie, corresponding to Lie subalgebra $T, Q_{1}$ is simply connected nilpotent normal subloop corresponding to the nilpotent ideal $N$. By Theorem 1 we have that $\hat{M}=S \oplus T \oplus N=N_{00}+M_{1}$, where $N_{00} \subseteq N$ is a nilpotent subalgebra and $M_{1}$ is an ideal of $\hat{M}$ that is contained in $\operatorname{Lie}(\hat{M})$. Since exponential map from $N$ to $Q_{1}$ is a bijection then $\operatorname{Exp}\left(N_{00}\right)=Q_{2} \subset Q_{1}$ is a nilpotent simply connected subloop of $\hat{G}$. Since $\hat{M}=N_{00}+M_{1}$ then $\hat{G}=Q_{2} \cdot G_{1}$, where $G_{1}$ is simply connected normal group Lie corresponding to the ideal $M_{1} \subset \operatorname{Lie}(\hat{M})$.

Since $\hat{M}$ is a splitting by Theorem 1 there exists a Malcev algebra $\tilde{M}=$ $N_{00} \oplus M_{1} \in N L_{k}$ and an epimorphism $\pi: \tilde{M} \longmapsto \hat{M}$.

Let $\tilde{G}$ be simply connected analytic Moufang loop corresponding to Malcev algebra $\tilde{M}$. Then $\tilde{G}=Q_{2} \times G_{1}$, where $Q_{2}$ and $G_{1}$ are subloops of $\hat{G}$. Notice that multiplication in $\tilde{G}$ may be given the following analogue of (3).

$$
\left(r_{1}, g_{1}\right) \cdot\left(r_{2}, g_{2}\right)=\left(r_{1} r_{2}, g_{1}^{r_{2}} g_{2}\right)
$$

Where $g_{1}^{r_{2}}=r_{2}^{-1} g_{1} r_{2}$ is natural action of Moufang loop $Q_{2}$ on the Lie group $G_{1}$ by automorphisms, since $A\left(Q_{2}\right)=J\left(N_{00}\right)$ acts trivially on $G_{1}$. Here $A\left(Q_{2}\right)$ is an associator of $Q_{2}$ and we used that $\left[J(\hat{M}), M_{1}\right] \subseteq[J(\hat{M}), \operatorname{Lie}(\hat{M})]=0$.

It is clear that $G_{1}$ is contained in the nucleus of $\tilde{G}$ and $\tilde{G} \in G_{k}$ if and only if $Q_{2} \in G_{k}$.

Lemma 6. Let $N$ be a nilpotent finite dimensional Malcev algebra and $R$ be the corresponding simply connected analytic Moufang loop.

If the corresponding local analytic Moufang loop $R_{\epsilon}$ satisfies some identity $f\left(x_{1}, \ldots, x_{n}\right)=1$, then the global analytic loop $R$ satisfies the same identity.

In particular, $N \in N L_{k}$ if and only if $R \in G_{k}$.

Proof. It is possible to assume that $R=N \simeq \mathbf{R}^{m}$ with multiplication (6). Let $f$ be an identity of the local analytic loop $R_{\epsilon}$. Then $f\left(x_{1}, \ldots, x_{n}\right)=\sum_{j} f_{j}$, where $f_{j}=f_{j}\left(x_{1}, \ldots, x_{n}\right)$ is a Lie word in $x_{1}, \ldots, x_{n}$. Let $v_{1}, \ldots, v_{m}$ be a basis of $N$. Then $f=\sum_{j=1}^{m} g_{j} v_{j}$, where $g_{j}=g_{j}\left(x_{1}, \ldots, x_{n}\right)$ is a polynomial function in $x_{1}, \ldots, x_{n}, j=1, \ldots, m$. Since $f$ is a local identity then $g_{j}=0$ if $\left|x_{s}\right|<\varepsilon$, $s=1, \ldots, n$ and $\varepsilon$ is small enough. But any polynomial function, which is equal 
to zero in some neighborhood of $\overline{0}$ is equal to zero for all values of the variables. Hence $f=1$ is an identity of the loop $R$.

Returning to the proof of the Theorem 2 we have that $\tilde{G} \in G_{k}$ due to Lemma 6. Hence $\hat{G} \in G_{k}$, since $\hat{G}$ is a homomorphic image of $\tilde{G}$.

With this the proof of the Theorem 2 is done.

\section{Acknowledgements}

The first author was supported by grants CNPq 307824/2016-0 and FAPESP 2018/ 23690-6. The second author was supported by grant FAPESP 2018/11292-

6. The third author thanks for the support to FAPESP for grant 2019/24418-0 and the University of Sao Paulo. The first and fourth authors was supported by UAEU UPAR grant G00002599.

\section{References}

[Fi] Filippov, V.T., Central simple Malcev algebras. (Russian)Algebra i Logika15(1976),no. 2,235242, 246.

[Gri1] Grishkov, A., An analogue of Levi 's theorem for Malcev algebras. Algebra and Logic 16 (1977), no. 4, 389396, 493.

[Gri2] Grishkov, A.,Decomposable Malcev algebras. Algebra and Logic 19 (1980), no. 4, 405422, 503.

[Car] Carlsson R., On the exceptional central simple non-Lie Malcev algebras, Trans. Amer. Math. Soc. 244 (1978), 173-184

[Ker] Kerdman, F.S. Analytic Moufang loops in the large, Algebra and Logic (1979) 18: 325. Translated from Algebra i Logika, Vol. 18, No. 5, pp. 523 - 555, September -October, (1979).

[Kuzm1] Kuz'min, E.N. Levi's theorem for Malcev algebras. (Russian) Algebra i Logika 16 (1977), no. 4, 424431, 493.

[Kuzm2] Kuz'min, E.N. On the relation between Mal'tsev algebras and analytic Mufang groups. Algebra and Logic (1971) 10: 1. Translated from Algebra i Logika, Vol. 10, No. 1, pp. 3 -22, January -February, (1971).

[Ma1] Malcev, A.I. Analytic loops. (Russian) Mat. Sb. N.S. 36(78), (1955). 569576 .

[Ma2] Malcev, A.I. On solvable Lie algebras. (Russian) Izv. Akad. Nauk SSSR Ser. Mat.9(1945), 329356 